\title{
The Construction of Rational Iterating Functions
}

\author{
By W. F. Smyth
}

\begin{abstract}
Suppose integers $n \geqslant 1$ and $\sigma \geqslant 2$ are given, together with $n$ distinct points $z_{1}, \ldots, z_{n}$, in the complex plane. Define $\Phi_{M}=\Phi_{M}\left(\sigma ; z_{1}, \ldots, z_{n}\right)$ to be the class of rational functions $\phi_{p, q}(z)=g_{p}(z) / h_{q}(z)$ (where $g$ and $h$ are polynomials of degree $p \geqslant 1$ and $q \geqslant 1$, respectively) such that (1) $p+q+1=M$, (2) $\phi$ when iterated converges with order $\sigma$ at each $z_{i}, i=1, \ldots, n$. Then if $M<\sigma n, \Phi_{M}$ is null; if $M=$ $\sigma n, \Phi_{M}$ contains exactly on elements. For every $M \geqslant \sigma n$, we show how to construct all the elements of $\Phi_{M}$ by expressing, for each choice of $p$ and $q$ which satisfies $p+$ $q+1=M$, the coefficients of $g_{p}$ and $h_{q}$ in terms of $M-\sigma n$ arbitrarily chosen values. In fact, these coefficients are expressed in terms of generalized Newton sums $S_{n}^{j, k}=$ $s_{n}^{j, k}\left(z_{1}, \ldots, z_{n}\right), 1 \leqslant j \leqslant n, k \geqslant n$, which we show may be calculated by recursion from the normal Newton sums $S_{n}^{j, n}$. Hence, given a polynomial $f_{n}(z)$ with $n$ distinct (unknown) zeros $z_{1}, \ldots, z_{n}$, we may construct all $\phi_{p, q}(z)$ which converge to the $z_{i}$ with order $\sigma:$ in the case $\sigma=2$, the choice $p=n, q=n-1$, yields the NewtonRaphson iteration $\phi_{n, n-1} \in \Phi_{2 n}$; the Schröder and König iterations are shown to be elements of $\Phi_{2(2 \sigma-3)(n-1)+2}$ and $\Phi_{2(\sigma-1)(n-1)+2}$, respectively. We show by example that there exist cases in which $\phi_{n, n-1}$ has an undesirable property (attractive cycles) not shared by other iterating functions in the same class $\Phi_{2 n}$.
\end{abstract}

Introduction. This research has its original motivation in the work of Julia [1], Fatou [2], and Montel [3] , [4] on the global convergence properties of iterations of meromorphic, particularly rational, functions. It appears that, for cases of practical interest, convergence of the sequence of iterates $z_{0}, \phi\left(z_{0}\right), \phi^{2}\left(z_{0}\right), \ldots$, is assured for every choice of starting point $z_{0}$ in the extended complex plane, except when $z_{0}$ is a point of a certain nowhere dense perfect set $E[5]$. Usually, of course, we have constructed $\phi$ in such a manner that the sequence of iterates will converge to a zero of a given function $f(z)$; that is, we have replaced the problem of finding $z$ such that $f(z)$ $=0$ by the problem of finding $z$ such that $\phi(z)=z$. (The most common form of $\phi$ is

$$
\phi(z)=z-f(z) / f^{\prime}(z),
$$

the Newton-Raphson iteration.) It may happen, however, that for choice of starting point $z_{0}$ in a certain domain, convergence takes place, not to a zero of $f(z)$, but to a set of $r \geqslant 2$ distinct points $\left(\alpha_{1}, \ldots, \alpha_{r}\right)$ such that

$$
\phi\left(\alpha_{1}\right)=\alpha_{2}, \ldots, \phi\left(\alpha_{r-1}\right)=\alpha_{r}, \quad \phi\left(\alpha_{r}\right)=\alpha_{1} ;
$$

so that, in fact, for each $k=1, \ldots, r, z=\alpha_{k}$ is a solution of $\phi^{r}(z)=z$. The bestknown example of this phenomenon is due to Turing and Brooker [6], who pointed

Received March 21, 1977; revised December 7, 1977.

AMS (MOS) subject classifications (1970). Primary 30A08, 40A05.

Copyright $\odot$ 1978, American Mathematical Society 
out that the Newton-Raphson iteration applied to the seemingly innocuous function

$$
f(z)=z^{4}-6 z^{2}-11
$$

gives rise to the attractive cycle $(+1,-1)$ for starting points $z_{0}$ in a certain neighborhood of either +1 or -1 .

Indeed, on the basis of the general theory of global convergence of rational functions, it is not surprising that such attractive cycles should sometimes exist. For let $g=g_{p}(z)$ and $h=h_{q}(z)$ be polynomials of degree $p$ and $q$, respectively, with no common factor. Then a basic result of the theory is that, in the immediate domain of convergence of each attractive fixpoint or attractive cycle of the rational iterating function

$$
\phi_{p, q}(z)=g_{p}(z) / h_{q}(z)
$$

there exists at least one critical point of the inverse iteration $\phi^{-1}$ [7]. Since these critical points are just the solutions of

$$
\phi^{\prime}(z)=\left(g^{\prime} h-g h^{\prime}\right) / h^{2}=0,
$$

we see that in general they shall be $p+q-1$ in number. In other words, convergence of the sequence of iterates of $\phi$ may take place to at most $p+q-1$ distinct attractive fixpoints or attractive cycles; the attractive fixpoints will be solutions of $\phi(z)=$ $z$, hence of $g-z h=0$, and the attractive cycles (if they exist) will be solutions of $\phi^{r}(z)=z$, for certain values of $r \geqslant 2$. In practice, as observed above, we shall have constructed $\phi(z)$ in such a manner that, of the $\max (p, q+1)$ fixpoints of $\phi(z)=z, n$ shall be attractive fixpoints and also solutions of a given equation $f(z)=0$. Hence, in general, there remains a possible total of $(p+q-1)-n$ attractive fixpoints or attractive cycles, to which convergence may take place from starting points in certain domains of the complex plane. In the case of the Newton-Raphson iteration, $p=n$, $q=n-1$, and we see that up to $n-2$ such cases may occur.

An ultimate aim of our research is therefore to find a means of constructing rational iterating functions which do not give rise to attractive cycles or superfluous attractive fixpoints, and which, therefore, converge globally-that is, almost everywhere in the complex plane-to solutions of the given problem $f(z)=0$. In this paper we take a step toward this goal by showing how to construct all the rational iterating functions $\phi=\phi_{p, q}$ which converge with given order of convergence $\sigma$ to $n$ given distinct points $z_{i}, i=1, \ldots, n$; in particular, how to construct all the $\phi_{p, q}$ which converge with order $\sigma$ to the distinct (but unknown) zeros $z_{i}$ of a given polynomial $f_{n}(z)$. Denote by

$$
\Phi_{M}=\Phi_{M}\left(\sigma ; z_{1}, \ldots, z_{n}\right)
$$

the class of rational functions $\phi_{p, q}$ such that (1) $p+q+1=M$, (2) the sequence of iterates of $\phi_{p, q}$ converges with order $\sigma$ at each $z_{i}$; then our construction technique opens up to us the new possibility of inspecting the iterating functions of each $\Phi_{M}$ to determine which give rise to attractive cycles, and which do not-or, indeed, to 
determine which may or may not have other desirable or undesirable properties. In this paper we do not propose any techniques for inspection of the elements of $\Phi_{M}$; but we do show, by an example derived from Brooker's, that, even though the NewtonRaphson iteration $\phi_{n, n-1} \in \Phi_{2 n}$ gives rise to attractive cycles, there may nevertheless exist other functions in the same class which have no such cycles.

\section{Rational Iterating Functions With $n$ Given Fixpoints. Consider}

$$
\phi_{p, q}(z)=g_{p}(z) / h_{q}(z)=\sum_{0 \leqslant j \leqslant p} \alpha_{j} z^{j} \mid \sum_{0 \leqslant k \leqslant q} \beta_{k} z^{k},
$$

where $p \geqslant 0, q \geqslant 0, \alpha_{p} \neq 0, \beta_{q} \neq 0$, and the polynomials $g$ and $h$ have no common factor. In this section we try to choose the coefficients $\left(\alpha_{j}, \beta_{k}\right)$ to satisfy $n$ given conditions

$$
\phi_{p, q}\left(z_{i}\right)=z_{i}, \quad i=1, \ldots, n,
$$

where $i \neq i^{\prime} \Rightarrow z_{i} \neq z_{i^{\prime}}$. That is, we must find $\left(\alpha_{j}, \beta_{k}\right)$ such that

$$
g\left(z_{i}\right)-z_{i} h\left(z_{i}\right)=0
$$

in other words, such that

$$
\begin{gathered}
\alpha_{0}+\left(\alpha_{1}-\beta_{0}\right) z_{i}+\cdots+\left(\alpha_{p}-\beta_{p-1}\right) z_{i}^{p}+\left(0-\beta_{p}\right) z_{i}^{p+1} \\
+\cdots+\left(0-\beta_{q}\right) z_{i}^{q+1}=0
\end{gathered}
$$

for each $z_{i}$, where if $q<p$, we define $\beta_{k} \equiv 0, k=q+1, \ldots, p$.

Setting

$$
\gamma_{j}^{(1)} \equiv \alpha_{j}-\beta_{j-1}, \quad j=0, \ldots, m,
$$

where $m \equiv \max (p, q+1)$, and defining $\beta_{-1} \equiv 0, \alpha_{j} \equiv 0$ for $j=p+1, \ldots, m$, we rewrite (3) as

$$
\left(\begin{array}{cccc}
1 & z_{1} & \cdots & z_{1}^{m} \\
\cdot & \cdot & & \cdot \\
\cdot & \cdot & & \cdot \\
\cdot & \cdot & & \cdot \\
1 & z_{n} & \cdots & z_{n}^{m}
\end{array}\right) \cdot\left(\begin{array}{c}
\gamma_{0}^{(1)} \\
\cdot \\
\cdot \\
\cdot \\
\gamma_{m}^{(1)}
\end{array}\right)=0
$$

Introducing the notation

$$
Z_{n}^{r, s} \equiv\left(\begin{array}{cccc}
z_{1}^{r} & z_{1}^{r+1} & \cdots & z_{1}^{s} \\
\cdot & \cdot & & \cdot \\
\cdot & \cdot & & \cdot \\
\cdot & \cdot & & \cdot \\
z_{n}^{r} & z_{n}^{r+1} & \cdots & z_{n}^{s}
\end{array}\right), \Gamma_{r, s} \equiv\left(\begin{array}{c}
\gamma_{r}^{(1)} \\
\cdot \\
\cdot \\
\cdot \\
\gamma_{s}^{(1)}
\end{array}\right)
$$


for integers $r$ and $s, 0 \leqslant r \leqslant s$, this becomes

$$
Z_{n}^{0, m} \Gamma_{0, m}=0
$$

a set of $n$ homogeneous linear equations in $m+1$ variables $\gamma_{j}^{(1)}$. We observe that for $m+1=n, \operatorname{det}\left(Z_{m+1}^{0, m}\right)$ is simply the Vandermonde determinant

$$
D_{n}=\prod_{n \geqslant i>i^{\prime} \geqslant 1}\left(z_{i}-z_{i^{\prime}}\right) \neq 0
$$

Accordingly, for $m<n$, the only solution of (5) is $\Gamma_{0, m}=0$, which implies $\alpha_{j}=$ $\beta_{j-1}, j=0, \ldots, m$, hence that $\phi_{p, q}(z)=z$, identically.

However, if we choose $m=n$, the rank of $Z_{n}^{0, m}$ is also $n$, and, therefore, (5) possesses a nontrivial solution in the $\gamma_{j}^{(1)}$ which is unique but for multiplication by a constant factor. Thus the minimum value of $m$ leading to a nontrivial iterating function $\phi_{p, q}$ is $m=n$. Rewriting (5) for $m=n$, we get

$$
Z_{n}^{0, n-1} \Gamma_{0, n-1}=-Z_{n}^{n, n} \gamma_{n}^{(1)}
$$

which yields the (unique) solution

$$
\gamma_{j}^{(1)}=-\left(D_{n}^{j+1, n} / D_{n}\right) \gamma_{n}^{(1)}, \quad j=0, \ldots, n-1,
$$

where in general $D_{n}^{j, k}$ is the determinant obtained by replacing the $j$ th column of $D_{n}$ with $Z_{n}^{k, k}$. In the Appendix we show (Theorem 5) that the quantities

$$
S_{n}^{j, n}=(-1)^{n-j} D_{n}^{j, n} / D_{n}, \quad 1 \leqslant j \leqslant n,
$$

are the familiar Newton sums

$$
S_{n}^{1, n}=z_{1} z_{2} \cdots z_{n}, \ldots, S_{n}^{n-1, n}=\sum_{i \neq i^{\prime}} z_{i} z_{i^{\prime}}, \quad S_{n}^{n, n}=\sum_{1 \leqslant i \leqslant n} z_{i}
$$

We write then for the case $m=n$ :

$$
\gamma_{j}^{(1)}=(-1)^{n-j} S_{n}^{j+1, n} \gamma_{n}^{(1)}, \quad j=0, \ldots, n-1,
$$

and we observe that the $\gamma_{j}^{(1)}$ are always symmetric functions of the $z_{i}$.

More generally, for $m \geqslant n$ conditions (1),

$$
Z_{n}^{0, n-1} \Gamma_{0, n-1}=-Z_{n}^{n, m} \Gamma_{n, m}
$$

and we see that each element of the solution $\Gamma_{0, n-1}$ may be expressed as a linear combination of $m-n+1$ arbitrarily chosen values $\gamma_{n}^{(1)}, \ldots, \gamma_{m}^{(1)}$. If corresponding to each $K=n, \ldots, m$, we choose these $m-n+1$ values so that $\gamma_{K}^{(1)} \neq 0 ; \gamma_{k}^{(1)}=$ 0 for $k \neq K$, we derive $m-n+1$ linearly independent particular solutions

$$
\gamma_{j K}^{(1)}=-\left(D_{n}^{j+1, K} / D_{n}\right) \gamma_{K}^{(1)}, \quad j=0, \ldots, n-1,
$$

each dependent on a single arbitrarily chosen value $\gamma_{K}^{(1)}$. We may then reconstitute a general solution of (7) by forming a linear combination of these $m-n+1$ particular solutions. Accordingly, summing the $\gamma_{j K}^{(1)}$ over $K$ and making use of the generalized Newton sums $S_{n}^{j, k}$ defined in the Appendix, we write the general solution of the system (5) as 


$$
\gamma_{j}^{(1)}=(-1)^{n-j} \sum_{n<K \leqslant m} S_{n}^{j+1, K} \gamma_{K}^{(1)}, \quad j=0, \ldots, n-1,
$$

where the $\gamma_{K}^{(1)}$ are arbitrary complex numbers, not all zero. The quantities $S_{n}^{j+1, K}$ are by definition symmetric functions of the $z_{i}$, and may be calculated as indicated by the Algorithm and Theorem 7 given in the Appendix. Hence we state

THEOREM 1. In order that a rational function $\phi_{p, q}(z)$ satisfy the $n$ conditions (1), it is necessary that $m \equiv \max (p, q+1) \geqslant n . \phi_{p, q}(z)$ satisfying (1) may be constructed by arbitrarily choosing $m-n+1$ "combined coefficients" $\gamma_{j}^{(1)}$ as defined in (4), and then using (8) or an analogous system to find the remaining $n$ combined coefficients, which are then uniquely determined as symmetric functions of the given points $z_{i}, i=1, \ldots, n$.

We have thus found a way to generate all rational functions which as iterating functions have fixpoints at $n$ given distinct points.

Rational Iterating Functions With $n$ Given Attractive Fixpoints. The conditions now to be satisfied may be expressed as follows:

$$
\phi_{p, q}\left(z_{i}\right)=z_{i}, \quad \phi_{p, q}^{\prime}\left(z_{i}\right)=\epsilon,
$$

for some $0 \leqslant|\epsilon|<1$ and every $i=1, \ldots, n$. These are $2 n$ independent simultaneous linear constraints on the $p+q+2$ coefficients $\left(\alpha_{j}, \beta_{k}\right)$, which we may reexpress in the form of (2):

$$
\begin{array}{r}
g\left(z_{i}\right)-z_{i} h\left(z_{i}\right)=0, \\
g^{\prime}\left(z_{i}\right)-z_{i} h^{\prime}\left(z_{i}\right)=\epsilon .
\end{array}
$$

From (10b) we obtain after some manipulation a set of equations analogous to (3):

$$
\sum_{0<j \leqslant m-1}\left[(j+1) \alpha_{j+1}-(j+\epsilon) \beta_{j}\right] z_{i}^{j}=0, \quad i=1, \ldots, n .
$$

It is convenient to define

$$
\gamma_{j}^{(2)}=(j+1) \alpha_{j+1}-(j+\epsilon) \beta_{j}, \quad j=0, \ldots, m-1,
$$

analogous to (4). Then (11) simplifies to

$$
\sum_{0<j \leqslant m-1} z_{i}^{j} \gamma_{j}^{(2)}=0, \quad i=1, \ldots, n,
$$

a set of $n$ homogeneous linear equations in $m$ unknowns $\gamma_{j}^{(2)}$ which is identical in form to (5). Proceeding as in the previous section, we observe that for $m<n+1$ there are only trivial solutions of (13), and that for $m=n+1$ there is a unique solution

$$
\gamma_{j}^{(2)}=(-1)^{n-j} S_{n}^{j+1, n} \gamma_{n}^{(2)}, \quad j=0, \ldots, n-1,
$$

analogous to (6). Then for $m \geqslant n+1$, we may show as before that general solutions $\gamma_{j}^{(2)}, j=0, \ldots, n-1$, of (10b) may be written 


$$
\gamma_{j}^{(2)}=(-1)^{n-j} \sum_{n \leqslant K \leqslant m-1} S_{n}^{j+1, K} \gamma_{K}^{(2)}, \quad j=0, \ldots, n-1
$$

identical in form to (8). The solutions (8) and (15) taken together are $2 n$ linear homogeneous equations in $p+q+2$ variables $\left(\alpha_{j}, \beta_{k}\right)$ equivalent to the original $2 n$ equations (9); they will possess a solution if $p+q+1 \geqslant 2 n$, a condition in some respects stricter than $m \geqslant n+1$, but which allows us to solve the equations (8) and (15) in the important "Newton-Raphson" case, characterized by $n=m=p=q$ +1 and $\epsilon=0$. We have then

THEOREM 2. In order that a rational function $\phi_{p, q}(z)$ satisfy the $2 n$ conditions (9), it is necessary that $p+q+1 \geqslant 2 n$. $\phi_{p, q}(z)$ satisfying (9) may be constructed by arbitrarily choosing $(p+q+2)-2 n$ of the coefficients $\left(\alpha_{j}, \beta_{k}\right)$, and then using (4), (8), (12), and (15) to find the remaining $2 n$ coefficients, which are then uniquely determined as symmetric functions of the given points $z_{i}, i=1, \ldots, n$.

We have thus found a way to generate all rational functions which as iterating functions have attractive fixpoints at $n$ given distinct points. If $\epsilon=0$, the generated iterating functions will have convergence of order 2 at each of the given points.

Rational Iterating Functions of Given Order $\sigma$. Evidently, the above results may be generalized. Given $n$ distinct points $z_{1}, \ldots, z_{n}$, and any integer $\sigma>1$, we may construct rational iterating functions $\phi_{p, q}$ with order of convergence $\sigma$ at each of the given points by finding solutions to the on linear homogeneous equations

$$
\begin{aligned}
\phi_{p, q}\left(z_{i}\right) & =z_{i}, \\
\phi_{p, q}^{(s-1)}\left(z_{i}\right) & =0, \quad s=2, \ldots, \sigma,
\end{aligned}
$$

where $i=1, \ldots, n$. If we introduce the notation $g^{(0)} \equiv g, h^{(0)} \equiv h$, we may briefly reexpress these equations in the form of $(10)$ :

$$
g^{(s-1)}\left(z_{i}\right)-z_{i} h^{(s-1)}\left(z_{i}\right)=0,
$$

for $s=1, \ldots, \sigma, i=1, \ldots, n$. If consistent with (4) and (12), we define

$$
\gamma_{j}^{(s)}=\left(\begin{array}{c}
j+s-1 \\
s-1
\end{array}\right) \alpha_{j+s-1}-\left(\begin{array}{c}
j+s-2 \\
s-1
\end{array}\right) \beta_{j+s-2},
$$

for $s=1, \ldots, \sigma, j=0, \ldots, m-s+1$, then we may rewrite (17) in the form

$$
\sum_{0 \leqslant j \leqslant m-s+1} z_{i}^{j} \gamma_{j}^{(s)}=0
$$

for $s=1, \ldots, \sigma, i=1, \ldots, n$, and, as before, derive general solutions

$$
\gamma_{j}^{(s)}=(-1)^{n-j} \sum_{n \leqslant K \leqslant m-s+1} S_{n}^{j+1, K} \gamma_{K}^{(s)}
$$

$s=1, \ldots, \sigma, j=0, \ldots, n-1$, valid provided $p+q+1 \geqslant \sigma n$. We have

THEOREM 3. In order that a rational function $\phi_{p, q}(z)$ satisfy the on conditions (16), it is necessary that $p+q+1 \geqslant \sigma n . \phi_{p, q}(z)$, satisfying (16), may be constructed 
by arbitrarily choosing $(p+q+2)$-on of the coefficients $\left(\alpha_{j}, \beta_{k}\right)$, and then using (18) and (20) to find the remaining on coefficients, which are then uniquely determined as symmetric functions of the given points $z_{i}, i=1, \ldots, n$.

In terms of the iteration classes $\Phi_{M}$ defined in the Introduction, Theorem 3 tells us that $\Phi_{M}$ is null whenever $M<\sigma n$. Moreover, for $M=\sigma n$, each of the $\sigma n$ possible choices of $p$ and $q$ yields a single unique iterating function $\phi_{p, q}$; in other words, $\Phi_{\sigma n}$ contains exactly on members. For $M>\sigma n$, each choice of $p$ and $q$ yields an infinite subclass of iterating functions with coefficients dependent on the particular choice of the excess $(p+q+2)$-on coefficients. The case $M=\sigma n$ is, therefore, of particular interest, not only because it is a minimum (hence minimizing time required for function evaluations, as well as number of possible attractive cycles), but also because $\Phi_{\sigma n}$ is finite.

Rational Iterating Functions for Zeros of a Polynomial $f_{n}(z)$. As mentioned. earlier, it is shown in the Appendix that the quantities $S_{n}^{j, k}=S_{n}^{j, k}\left(z_{1}, \ldots, z_{n}\right)$ used to compute the coefficients of $\phi_{p, q}$ may themselves be computed from the Newton sums. Since the Newton sums may in their turn be computed from the coefficients only of a polynomial [8, p. 453], without direct knowledge of the roots, it is natural to seek to apply the method to construct rational iterating functions convergent to the unknown (but distinct) zeros of a given polynomial $f_{n}(z)$. Such an application is straightforward, and an example is provided in the next section.

In this section we establish a connection between our iteration classes $\Phi_{M}$ and one or two well-known iterating functions (or classes of iterating functions) which are applied to the zeros of polynomials. We observe first that for $\sigma=2$ the iterating function $\phi_{n, n-1} \in \Phi_{2 n}\left(2 ; z_{1}, \ldots, z_{n}\right)$ is exactly the Newton-Raphson iteration applied to a polynomial $f_{n}(z)$ with $n$ distinct roots $z_{1}, \ldots, z_{n}$. This result is a direct consequence of the uniqueness of $\phi_{n, n-1}$ (Theorem 3), but may also be proved directly.

A general class of iterations was discovered by Eggers and Schröder [9] about a century ago and has been connected in a recent paper [10] to a number of more modern iterations. Given a polynomial $f_{n}(z)$ of degree $n$, Schröder described a method of finding a rational iterating function of any desired order of convergence $\sigma>1$ convergent to the zeros of $f_{n}$; his iterations took the form

$$
\phi^{[\sigma]}(z)=z-b_{1}(z) f_{n}(z)+\cdots+(-1)^{\sigma-1} b_{\sigma-1}(z)\left[f_{n}(z)\right]^{\sigma-1},
$$

where $b_{1}(z)=1 / f^{(1)}(z), b_{s}(z)=b_{s-1}^{(1)}(z) /\left[s f_{n}^{(1)}(z)\right], s=2, \ldots, \sigma-1$, and turn out to have numerator of degree $(2 \sigma-3)(n-1)+1$ and denominator of degree one less (they are Newton-Raphson-like in form). In our terminology Schröder's $\phi^{[\sigma]}(z)$ is one of the infinitely many iterations

$$
\phi_{(2 \sigma-3)(n-1)+1,(2 \sigma-3)(n-1)} \in \Phi_{2(2 \sigma-3)(n-1)+2},
$$

and we see that $\phi^{[\sigma]}$ actually depends upon an implicit arbitrary choice of $2(2 \sigma-3)(n-1)+2-\sigma n$ or $(\sigma-2)(3 n-4)$ coefficients. For $\sigma=2, \phi^{[2]}=$ $\phi_{n, n-1}$. 
Householder [11, p. 103] describes another general class of iterations due to König, which are also designed to converge with order $\sigma$ to the zeros of polynomial $f_{n}$. König's iteration takes the form

$$
\psi^{[\sigma]}(z)=z+(\sigma-1)\left[1 / f_{n}(z)\right]^{(\sigma-2)} /\left[1 / f_{n}(z)\right]^{(\sigma-1)}
$$

is also Newton-Raphson-like, and has numerator of degree $(\sigma-1)(n-1)+1$. $\psi^{[\sigma]}(z)$ is one of the infinitely many iterations

$$
\phi_{(\sigma-1)(n-1)+1,(\sigma-1)(n-1)} \in \Phi_{2(\sigma-1)(n-1)+2}
$$

and depends upon an implicit arbitrary choice of $(\sigma-2)(n-2)$ coefficients.

Application to $f_{4}(z)=z^{4}-4 z^{2}-5$. This polynomial, suggested by Brooker's example [6], shares the property that the Newton-Raphson iteration applied to it gives rise to an attractive cycle. It has zeros $\pm \sqrt{5}$ and $\pm i$, and the Newton sums may be read off from the coefficients :

$$
S_{4}^{1,4}=-5, \quad S_{4}^{2,4}=0, \quad S_{4}^{3,4}=-4, \quad S_{4}^{4,4}=0 .
$$

With these values as our only input data, and making use of Theorem 3 and the Algorithm and results developed in the Appendix, we generate all the rational iterating functions $\phi_{p, q}$ which converge with order $\sigma=2$ to the zeros of $f_{4}$ and which belong to the minimum class $\Phi_{8}(2 ;+\sqrt{5},-\sqrt{5},+i,-i)$. Table 1 displays the results : observe that while $\phi_{4,3}$, for example, gives rise to the attractive cycle $(+\sqrt{5 / 7},-\sqrt{5 / 7})$, the iterations $\phi_{2,5}, \phi_{6,1}, \phi_{1,6}$, and $\phi_{7,0}$ converge to some root of $f_{4}$ from each critical point.

\section{TABLE 1}

\section{Iterating functions for $f_{4}(z)=z^{4}-4 z^{2}-5$ which belong to} minimum class $\Phi_{8}(2 ;+\sqrt{5},-\sqrt{5},+i,-i)$

$\begin{array}{cccllc}m & p & q & \text { iterating function } \phi_{p, q} & \begin{array}{c}\text { additional } \\ \text { critical points } \\ \text { (cps)* }\end{array} & \begin{array}{c}\text { fixpoints or cycles } \\ \text { reached from cps }\end{array} \\ 4 & 4 & 3 & \left(3 z^{4}-4 z^{2}+5\right) /\left(4 z^{3}-8 z\right) & \pm \sqrt{2 / 3} & (+\sqrt{5 / 7},-\sqrt{5 / 7}) \\ 5 & 3 & 4 & \left(8 z^{3}+20 z\right) /\left(z^{4}+4 z^{2}+15\right) & \pm \sqrt{15 / 2} i & (+\sqrt{7} i,-\sqrt{7} i) \\ 5 & 5 & 2 & \left(z^{5}-12 z^{3}-25 z\right) /\left(-8 z^{2}-20\right) & \pm(5 / \sqrt{6}) i & \infty \\ 6 & 2 & 5 & \left(92 z^{2}-40\right) /\left(3 z^{5}-20 z^{3}+109 z\right) & \pm \sqrt{218 / 207} i & \pm i \\ 6 & 6 & 1 & \left(z^{6}-6 z^{4}-33 z^{2}+10\right) /(-36 z) & \pm \sqrt{2 / 5 i} & \pm i \\ 7 & 1 & 6 & -180 z /\left(2 z^{6}-21 z^{4}+42 z^{2}-115\right) & \pm \sqrt{23 / 10} & \pm \sqrt{5} \\ 7 & 7 & 0 & \left(2 z^{7}-21 z^{5}+42 z^{3}+245 z\right) / 180 & \pm \sqrt{7 / 2} & \pm \sqrt{5} \\ 8 & 0 & 7 & -900 /\left(23 z^{7}-174 z^{5}+33 z^{3}+1130 z\right) & \pm \sqrt{226 / 161} & (+0.9285,-0.9285)\end{array}$

*The roots $\pm \sqrt{5}, \pm i$ are critical points of each $\phi_{p, q}^{-1}$. Thus, in each case, two other critical points remain to be specified, and these may possibly belong to the immediate domain of convergence of an attractive cycle, if such exist, or of a root. Note that "convergence" to $\infty$ is possible. 
Table 2 displays 5 of the 12 iterating functions in $\Phi_{12}(3 ;+\sqrt{5},-\sqrt{5},+i,-i)$; that is, the minimal class of rational iterating functions which converge with order $\sigma=3$ to the zeros of $f_{4}$.

\section{TABLE 2}

Iterating functions for $f_{4}(z)$ which belong to $\Phi_{12}(3 ;+\sqrt{5},-\sqrt{5},+i,-i)$

$$
\begin{array}{cccc}
m & p & q & \text { iterating function } \phi_{p, q} \\
6 & 6 & 5 & \left(49 z^{6}-12 z^{4}+675 z^{2}-200\right) /\left(87 z^{5}-204 z^{3}+645 z\right) \\
7 & 5 & 6 & \left(129 z^{5}+204 z^{3}+435 z\right) /\left(8 z^{6}+135 z^{4}+12 z^{2}+245\right) \\
7 & 7 & 4 & \left(8 z^{7}-231 z^{5}-252 z^{3}-805 z\right) /\left(-273 z^{4}+84 z^{2}-435\right) \\
8 & 4 & 7 & \left(1443 z^{4}-8220 z^{2}+3225\right) /\left(49 z^{7}-576 z^{5}+3363 z^{3}-8900 z\right) \\
8 & 8 & 3 & \left(29 z^{8}-280 z^{6}-1050 z^{4}+4760 z^{2}-2275\right) /\left(-2016 z^{3}+5760 z\right)
\end{array}
$$

The determination of the coefficients $\left(\alpha_{j}, \beta_{k}\right)$ for this example involved the solution of the simultaneous equations (20) in accordance with Theorem 3. This solution was facilitated by the fact that these equations are sparse in the $\left(\alpha_{j}, \beta_{k}\right)$; and an estimate of the maximum density of the matrix representing the equations may, therefore, be of general interest. (20) represents on equations in $p+q+2$ variables. The lefthand side of (20) specifies at most $2 \sigma n$ nonzero coefficients $\left(\alpha_{j}, \beta_{k}\right)$, hence represents at most that many matrix positions. The right-hand side specifies at most $\Sigma_{1 \leqslant s \leqslant \sigma}(m-s+1-n+1)$ nonzero coefficients. Maximum nonzero entries in the coefficient matrix are, therefore,

$$
2 \sigma n+\sum(m-s-n+2)=(\sigma / 2)(2 n+2 m-\sigma+3),
$$

out of a total $\sigma n(p+q+2)$ positions. The maximum ratio of nonzero positions in the matrix representation of the Eqs. (20) is, therefore,

$$
(2 n+2 m-\sigma+3) / 2 n(p+q+2) .
$$

If we let $m$ take its maximum value on, and confine ourselves to the "minimal" case $p+q+1=n$, this ratio becomes after some manipulation

$$
1 / n+(2 n-\sigma+1) / 2 n(\sigma n+1)
$$

which, since $\sigma \geqslant 2$, is less than $3 / 2 n$. The system (20) will thus, in general, be fairly sparse.

Extension of Results to Multiple Fixpoints. In the preceding development it has most obviously been the definition of $S_{n}^{j, k}$ in terms of an expression with denominator $D_{n}$ (vanishing whenever $z_{i}=z_{i^{\prime}}, i \neq i^{\prime}$ ) which has precluded consideration of multiple fixpoints. In this section it will be found that the vanishing of $D_{n}$ is not in itself a fundamental problem, but that other difficulties, similar to those encountered when applying the Newton-Raphson iteration to a function with multiple zeros, inhibit full extension of the results to the multiple fixpoint case. 
We begin by considering Eq. (2), which, it has been remarked [12], expresses the condition on $g(z)-z h(z)$, a polynomial of degree $m$, that it have $n \leqslant m$ roots $z_{i}$; that is, that

$$
\begin{aligned}
{\left[\sum_{0 \leqslant j \leqslant m} \gamma_{j}^{(1)} z^{j}\right] } & =g(z)-z h(z) \\
& =C(z) \prod_{1 \leqslant i \leqslant n}\left(z-z_{i}\right)=\left[C(z) \sum_{0 \leqslant i \leqslant n}(-1)^{n-i} S_{n}^{i+1, n} z^{i}\right],
\end{aligned}
$$

where the $S_{n}^{i+1, n}$ are as before simply the Newton sums, $S_{n}^{n+1, n} \equiv 1$, and

$$
C(z)=c_{n}+c_{n+1} z+\cdots+c_{m-1} z^{m-n-1}+c_{m} z^{m-n}, \quad c_{m} \neq 0,
$$

is a polynomial with arbitrary coefficients introduced so that the bracketed polynomials should be of equal degree $m$. We recall, however, that some of the left-hand coefficients-to be precise, $\gamma_{K}^{(1)}, K=n, \ldots, m$-may also be arbitrarily chosen; and we realize then that we may either express the $\gamma_{K}^{(1)}$ in terms of the $c_{K}, K=n, \ldots$, $m$, or vice versa, simply by equating the coefficients of like powers of $z$. We find then

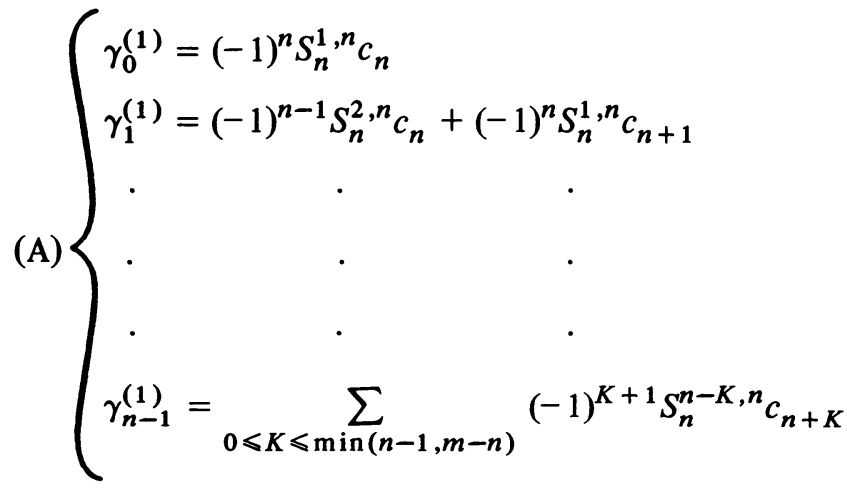

$$
\begin{aligned}
& \text { (B) }\left\{\begin{array}{l}
\gamma_{n}^{(1)}=\sum_{0 \leqslant K \leqslant \min (n, m-n)}(-1)^{K} S_{n}^{n-K+1, n} c_{n+K} \\
\cdot \\
\cdot \\
\gamma_{m-1}^{(1)}=S_{n}^{n+1, n} c_{m-n-1}+(-1) S_{n}^{n, n} c_{m-n} \\
\gamma_{m}^{(1)}=S_{n}^{n+1, n} c_{m-n} .
\end{array}\right.
\end{aligned}
$$

We may imagine, first of all, solving (B) for the $c_{K}$ in terms of the $\gamma_{K}^{(1)}, K=$ $n, \ldots, m$; this we may clearly accomplish by a process of back substitution. Then, if we substitute the resulting expressions for the $c_{K}$ into (A), we shall be able to write down solutions which express the $\gamma_{j}^{(1)}, j=0, \ldots, n-1$, in terms of the $\gamma_{K}^{(1)}$, $k=n, \ldots, m$, and the Newton sums, just as in the preceding sections, but this time avoiding the use of Vandermonde determinants. We do not need actually to carry out this complex calculation, however, since we already know, from our previous 
work, the result of it. For distinct $z_{i}$, we shall certainly find, as before, that

$$
\gamma_{j}^{(1)}=(-1)^{n-j} \sum_{n \leqslant K \leqslant m} S_{n}^{j+1, K} \gamma_{K}^{(1)}, \quad j=0, \ldots, n-1,
$$

but with the important difference now that the values $S_{n}^{j+1, K}$ are no longer defined in terms of Vandermonde determinants, but may now be regarded as defined by the relation (30), found in the Appendix, which states that for $k \geqslant n \geqslant 1$ and $1 \leqslant j \leqslant n$

$$
S_{n}^{j, k}=\sum_{0 \leqslant j^{\prime} \leqslant j-1}(-1)^{j^{\prime}} S_{n}^{n, k-j^{\prime}-1} S_{n}^{j-j^{\prime}, n},
$$

with the particular case

$$
S_{n}^{n, k}=\sum_{0 \leqslant j^{\prime} \leqslant n-1}(-1)^{j^{\prime}} S_{n}^{n, k-j^{\prime}-1} S_{n}^{n-j^{\prime}, n}
$$

which allows us, using the Algorithm given in the Appendix, to recursively determine the $S_{n}^{n, k}$, hence the $S_{n}^{j, k}$, from the Newton sums and $S_{n}^{n, n-1} \equiv 1$. Based on this second definition of the $S_{n}^{j+1, K}$, these calculations may now equally be carried out for the case of multiple $z_{i}$; and they will, by the continuity of $\gamma_{j}^{(1)}\left(z_{1}, \ldots, z_{n}\right)$, yield the same result (8). We state then

THEOREM 1'. Theorem 1 holds also in the case that the $n$ given fixpoints $z_{i}$ are not necessarily distinct.

Difficulties arise, however, when we try to extend Theorems 2 and 3 to the case of multiple fixpoints. For suppose $z_{i}$ is a fixpoint of exact multiplicity $a \geqslant 1$. Then

$$
g(z)-z h(z)=\left(z-z_{i}\right)^{a} A(z),
$$

for some polynomial $A(z)$ such that $A\left(z_{i}\right) \neq 0$. Since

$$
g^{\prime}(z)-z h^{\prime}(z)=a\left(z-a_{i}\right)^{a-1} A(z)+\left(z-z_{i}\right)^{a} A^{\prime}(z)+h(z)
$$

and

$$
\phi^{\prime}(z)=\left[g^{\prime}(z)-\phi(z) h^{\prime}(z)\right] / h(z),
$$

we conclude that if $\left(z-z_{i}\right)^{a-1}$ is not a factor of $h(z)$,

$$
\begin{aligned}
\phi^{\prime}\left(z_{i}\right) & =\lim _{z \rightarrow z_{i}}\left[a\left(z-z_{i}\right)^{a-1} A(z)+\left(z-z_{i}\right)^{a} A^{\prime}(z)+h(z)-(\phi(z)-z) h^{\prime}(z)\right] / h(z) \\
& =1,
\end{aligned}
$$

so that $z_{i}$ cannot be an attractive, but is rather an indifferent [13], fixpoint of $\phi$. In this case, then, Eqs. (10) and (17) are both internally inconsistent, and we cannot expect a meaningful solution.

Let us suppose then that $\left(z-z_{i}\right)^{a-1}$ is a factor of $h(z)$, and that $a>1$. Then $h(z)=\left(z-z_{i}\right)^{a-1} B(z)$, for some polynomial $B(z)$, and

$$
\phi(z)=\left(z-z_{i}\right)^{a-1}\left[z B(z)+\left(z-z_{i}\right) A(z)\right] /\left(z-z_{i}\right)^{a-1} B(z),
$$


which, since our construction process makes no provision for cancellation of identical factors in numerator and denominator, is of the form $0 / 0$ at $z=z_{i}$. Furthermore, $\phi^{\prime}(z)$ is of the same form $0 / 0$ at $z=z_{i}$, and we conclude that in this case also multiple fixpoints introduce internal inconsistencies into Eqs. (10) and (17).

It becomes clear then that for multiple fixpoints we require an improved construction process which will somehow cancel the terms $\left(z-z_{i}\right)^{a-1}$ to yield

$$
\psi(z)=z+\left(z-z_{i}\right) A(z) / B(z) \text { and }\left.\quad \psi^{\prime}(z)\right|_{z=z_{i}}=1+A\left(z_{i}\right) / B\left(z_{i}\right)
$$

effectively reducing the problem to the case of distinct fixpoints, and enabling us to solve (10) and (17) as before.

It is nevertheless interesting to see what happens when the equations (10) or (17) are applied to a particular example of multiple fixpoints. The results of such an application are displayed in Table 3. Provided both $g_{p}(z)$ and $h_{q}(z)$ are of degree sufficiently large to contain a factor $\left(z-z_{i}\right)^{a-1}$, it seems that the iterating function $\psi_{p, q}$ may be used to solve the given problem, but with a lower order of convergence at multiple fixpoints $z_{i}$. The case in which $\left(z-z_{i}\right)^{a-1}$ does not divide $h(z)$ apparently does not occur. The repeated result $\psi^{\prime}(1)=1 / 2$ suggests a possible generalization of the well-known fact $[14$, p. 53] that for the Newton-Raphson iteration, at a fixpoint $z_{i}$ of multiplicity $a$,

$$
\phi^{\prime}\left(z_{i}\right)=1-1 / a
$$

This typical reduction of the order of convergence at multiple fixpoints is a problem not only for iterations of the form treated in this paper-that is, $z^{(j+1)}=\phi\left(z^{(j)}\right)-$ but also for more general forms of iteration: an analysis of the local convergence properties of one such iterative form, using some techniques similar to those employed here and including the case of multiple roots, is presented in [15].

REMARK. The method described here provides a means of constructing and classifying rational iterating functions of specified order of convergence. It would be valuable to extend these results so as to be able to construct efficiently an iterating function for a given problem which in addition possesses desirable convergence characteristics.

Acknowledgement. I should like to thank James L. Howland, University of Ottawa, for introducing me to this topic and assisting me in the early stages of this research.

\section{Appendix}

Given integers $n \geqslant 1, j$, and $k$, and distinct (real or complex) values $z_{i}, i=1$, $\ldots, n$, we define quantities $S_{n}^{j, k}=S_{n}^{j, k}\left(z_{1}, \ldots, z_{n}\right)$ as follows:

$$
\begin{aligned}
S_{n}^{j, k} & =(-1)^{n-j} D_{n}^{j, k} / D_{n} \quad \text { for } 1 \leqslant j \leqslant n \text { and } k \geqslant 0 \\
& =0 \quad \text { otherwise, }
\end{aligned}
$$

where $D_{n}=D_{n}\left(z_{1}, \ldots, z_{n}\right)$ is the Vandermonde determinant of order $n$, 


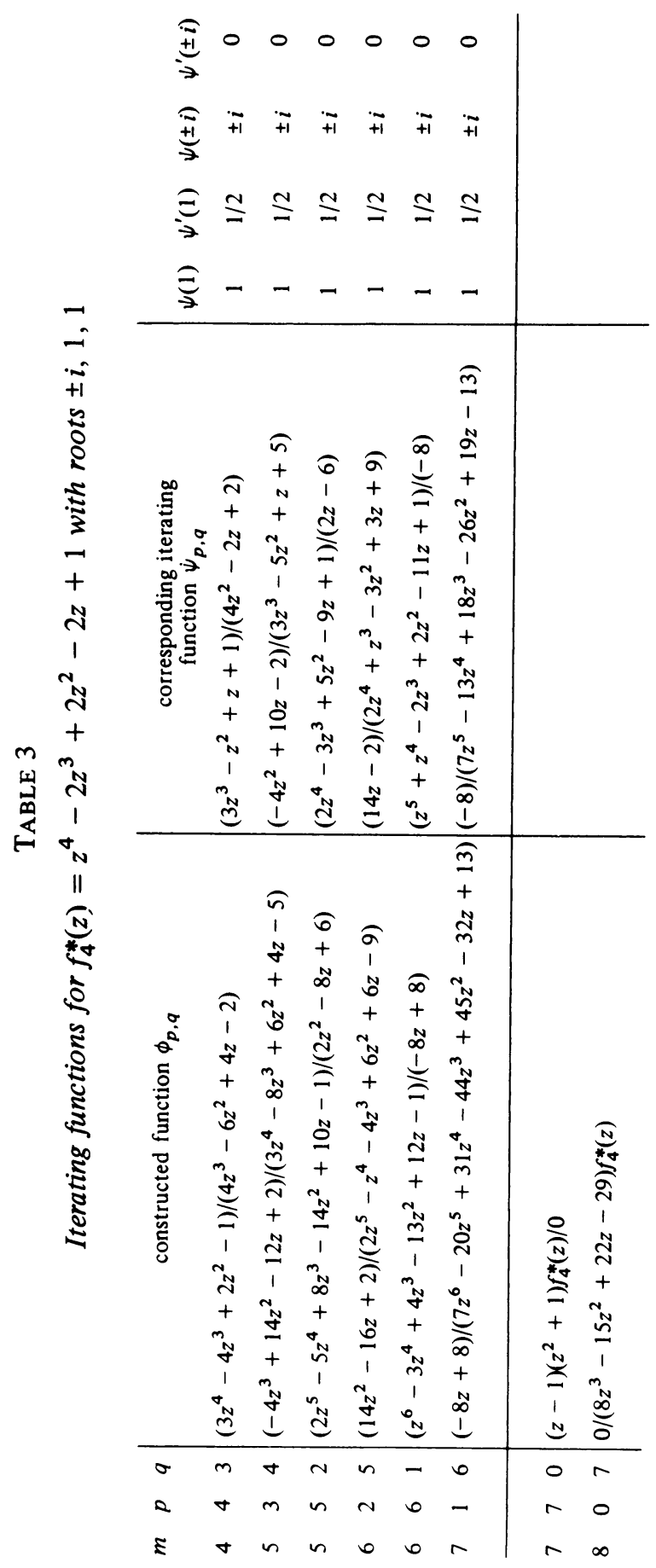




$$
\left|\begin{array}{cccc}
1 & z_{1} & \cdots & z_{1}^{n-1} \\
1 & z_{2} & \cdots & z_{2}^{n-1} \\
\cdot & \cdot & \cdot \\
\cdot & \cdot & \cdot \\
\cdot & \cdot & \cdot \\
1 & z_{n} & \cdots & z_{n}^{n-1}
\end{array}\right|,
$$

and $D_{n}^{j, k}$ is the determinant obtained by replacing the $j$ th column of $D_{n}$, which we denote $\left(z_{i}^{j-1}\right)$, by the new column $\left(z_{i}^{k}\right)$. Then, in particular, $D_{1}=1$ and $S_{1}^{1, k}=$ $D_{1}^{1, k}=z_{1}^{k}$. For $n \geqslant 2$, recall that

$$
D_{n}=\Pi_{n} D_{n-1}
$$

where $\Pi_{n}=\Pi_{1 \leqslant i \leqslant n-1}\left(z_{n}-z_{i}\right)$, from which it follows that

$$
D_{n}=\prod_{1 \leqslant i<i^{\prime} \leqslant n}\left(z_{n}-z_{i}\right)
$$

Note also that when $k \leqslant n-1$,

$$
\begin{aligned}
D_{n}^{j, k} & =D_{n} \quad \text { for } j=k+1 ; \\
& =0 \quad \text { otherwise. }
\end{aligned}
$$

THEOREM 4. For $k \geqslant n \geqslant 2$,

$$
S_{n}^{j, k}=\sum_{1 \leqslant j^{\prime} \leqslant k-n+1} S_{n-1}^{j, k-j^{\prime}} z_{n}^{j^{\prime}}+\sum_{0 \leqslant j^{\prime} \leqslant k-n+1} S_{n-1}^{j-1, k-j^{\prime}-1} z_{n}^{j^{\prime}} .
$$

Proof. The result is trivially true for $j<1$ and for $j>n$. For $j=n$,

$$
D_{n}^{n, k}=\left|\begin{array}{cccc}
1 & z_{i} \cdots z_{1}^{n-2} & z_{1}^{k} \\
\cdot & \cdot & \cdot & \cdot \\
\cdot & \cdot & \cdot & \cdot \\
\cdot & \cdot & \cdot & \cdot \\
1 & z_{n} \cdots & z_{n}^{n-2} & z_{n}^{k}
\end{array}\right| .
$$

Subtracting the $n$th row of this determinant from each of the others, and then extracting the common factor $\Pi_{n}$, we find that $D_{n}^{n, k}$ is equal to $(-1)^{n-1}(-1)^{n-1} \Pi_{n}$ times an $n-1$ by $n-1$ determinant whose $h$ th column, $1 \leqslant h \leqslant n-2$, may be represented

$$
\left(\sum_{1 \leqslant j^{\prime} \leqslant h} z_{n}^{h-j^{\prime}} z_{i}^{j^{\prime}-1}\right)
$$

and whose $(n-1)$ th column is

$$
\left(\sum_{1 \leqslant j^{\prime} \leqslant k} z_{n}^{k-j^{\prime}} z_{i}^{j^{\prime}-1}\right) .
$$

Then, subtracting $z_{n}^{k-n+2}$ times the $(n-2)$ th column from the $(n-1)$ th column, and 
afterwards successively subtracting $z_{n}$ times the $(h-1)$ th column from the $h$ th column $h=n-2, \ldots, 2$, we find

$$
\begin{aligned}
D_{n}^{n, k}=\Pi_{n}\left|\begin{array}{llll}
1 & z_{1} \cdots z_{1}^{n-2} & \sum_{0 \leqslant j^{\prime} \leqslant k-n+1} z_{n}^{j^{\prime}} z_{1}^{k-j^{\prime}-1} \\
\cdot & \cdot & \cdot \\
\cdot & \cdot \\
1 & z_{n-1} \cdots & z_{n-1}^{n-2} \sum_{0 \leqslant j^{\prime} \leqslant k-n+1} z_{n}^{j^{\prime}} z_{n-1}^{k-j^{\prime}-1}
\end{array}\right| \\
=\Pi_{n} \sum_{0 \leqslant j^{\prime} \leqslant k-n+1} D_{n-1}^{n-1, k-j^{\prime}-1} z_{n}^{j^{\prime}},
\end{aligned}
$$

from which, in view of (21) and the definition of $S_{n}^{j, k}$, the result follows immediately. Similar manipulations establish the theorem in the case $1 \leqslant j<n$.

We note some special cases of Theorem 4. For $j=1$,

$$
S_{n}^{1, k}=\sum_{1 \leqslant j^{\prime} \leqslant k-n+1} S_{n-1}^{1, k-j^{\prime}} z_{n}^{j^{\prime}}
$$

and for $j=n$,

$$
S_{n}^{n, k}=\sum_{0 \leqslant j^{\prime} \leqslant k-n+1} S_{n-1}^{n-1, k-j^{\prime}-1} z_{n}^{j^{\prime}}
$$

For $k=n$,

$$
S_{n}^{j, n}=S_{n-1}^{j, n-1} z_{n}+S_{n-1}^{j-1, n-1}
$$

whence

$$
\begin{aligned}
& S_{n}^{1, n}=S_{n-1}^{1, n-1} z_{n}, \\
& S_{n}^{n, n}=S_{n-1}^{n-1, n-1}+z_{n},
\end{aligned}
$$

and the following theorem may easily be proved by induction.

THEOREM 5. For $1 \leqslant j \leqslant n$ the quantities $S_{n}^{j, n}\left(z_{1}, \ldots, z_{n}\right)$ are the Newton sums for a polynomial of degree $n$ with distinct zeros $z_{i}, i=1, \ldots, n$; that is, $S_{n}^{j, n}$ is the sum of all distinct products of the $z_{i}$ taken $n-j+1$ at a time.*

Theorem 5 establishes then the important fact that given any polynomial $f_{n}(z)$ of degree $n$, we may determine corresponding quantities $S_{n}^{j, n}$ computed only from the coefficients of $f_{n}$. We go on now to show that in fact every $S_{n}^{j, k}$ may be computed from the $S_{n}^{j, n}$, hence also from the coefficients of $f_{n}$.

THEOREM 6. For $k \geqslant n \geqslant 1$ and $1 \leqslant j \leqslant n$,

$$
S_{n}^{j, k}+S_{n}^{j-1, k-1}=S_{n}^{n, k-1} S_{n}^{j, n} .
$$

\footnotetext{
*Using Theorem 4, it may also be shown more generally that for $1 \leqslant j \leqslant n$ and $k \geqslant n$, $s_{n}^{j, k}$ is the sum of all distinct products $z_{a}^{a^{\prime}} z_{b}^{b^{\prime}} \ldots$, where $\max \left(a^{\prime}, b^{\prime}, \ldots\right)=k-n+1$ and $a^{\prime}+$ $b^{\prime}+\ldots=k-j+1$.
} 
Proof. Using the definition of $S_{n}^{j, k}$, we may easily verify this result for $n=1$. Suppose we know that

$$
S_{n-1}^{j-1, k}+S_{n-1}^{j-2, k-1}=S_{n-1}^{n-1, k-1} S_{n-1}^{j-1, n-1}
$$

for all $k \geqslant n-1 \geqslant 1$ and $1 \leqslant j \leqslant n$. Then

$$
\begin{aligned}
S_{n}^{j, k}+S_{n}^{j-1, k-1}= & \sum_{1 \leqslant j^{\prime} \leqslant k-n+1} S_{n-1}^{j, k-j^{\prime} z_{n}^{j^{\prime}}+} \sum_{0<j^{\prime}<k-n+1} S_{n-1}^{j-1, k-j^{\prime}-1} z_{n}^{j^{\prime}} \\
& +\sum_{1 \leqslant j^{\prime} \leqslant k-n} S_{n-1}^{j-1, k^{\prime}-j^{\prime}-1} z_{n}^{j^{\prime}}+\sum_{0 \leqslant j^{\prime} \leqslant k-n} S_{n-1}^{j-2, k-j^{\prime}-2} z_{n}^{j^{\prime}} \\
= & \sum_{1 \leqslant j^{\prime}<k-n+1}\left\{S_{n-1}^{j, k-j^{\prime}}+S_{n-1}^{j-1, k-j^{\prime}-1}\right\} z_{n}^{j^{\prime}} \\
& +\sum_{0 \leqslant j^{\prime} \leqslant k-n}\left\{S_{n-1}^{j-1, k-j^{\prime}-1}+S_{n-1}^{j-2, k-j^{\prime}-2}\right\} z_{n}^{j^{\prime}} \\
& -S_{n-1}^{j-1, n-2} z_{n}^{k-n+1}+S_{n-1}^{j-1, n-2} z_{n}^{k-n+1} \\
= & \sum_{1 \leqslant j^{\prime}<k-n+1} S_{n-1}^{n-1, k-j^{\prime}-1} S_{n-1}^{j, n-1} z_{n}^{j^{\prime}} \\
& +\sum_{0 \leqslant j^{\prime} \leqslant k-n} S_{n-1}^{n-1, k-j^{\prime}-2} S_{n-1}^{j-1, n-1} z_{n}^{j^{\prime}},
\end{aligned}
$$

by the inductive hypothesis, if $j \neq n$; then by (25) and (26),

$$
\begin{aligned}
S_{n}^{j, k}+S_{n}^{j-1, k-1} & =\sum_{0 \leqslant j^{\prime} \leqslant k-n} S_{n-1}^{n-1, k-j^{\prime}-2} z_{n}^{j^{\prime}}\left\{z_{n} S_{n-1}^{j, n-1}+S_{n-1}^{j-1, n-1}\right\} \\
& =S_{n}^{j, n} S_{n}^{n, k-1} .
\end{aligned}
$$

If $j=n$,

$$
\begin{aligned}
S_{n}^{n, k}+S_{n}^{n-1, k-1} & =\sum_{1<j^{\prime} \leqslant k-n+1} S_{n-1}^{n-1, k-j^{\prime}-1} z_{n}^{j^{\prime}} \sum_{0<j^{\prime} \leqslant k-n} S_{n-1}^{n-1, k-j^{\prime}-2} z_{n}^{j^{\prime}} \\
& =\sum_{0 \leqslant j^{\prime} \leqslant k-n} S_{n-1}^{n-1, k-j^{\prime}-1}\left\{z_{n}+S_{n-1}^{n-1, n-1}\right\}=S_{n}^{n, k-1} S_{n}^{n, n},
\end{aligned}
$$

by application of (25) and (28). This completes the proof.

An immediate consequence of Theorem 6 is

THEOREM 7. For $k \geqslant n \geqslant 1$ and $1 \leqslant j \leqslant n$,

$$
S_{n}^{j, k}=\sum_{0 \leqslant j^{\prime} \leqslant j-1}(-1)^{j^{\prime}} S_{n}^{n, k-j^{\prime}-1} S_{n}^{j-j^{\prime}, n} .
$$

Theorem 7 shows that the determination of the $S_{n}^{j, k}$ may be reduced to the determination of the Newton sums $S_{n}^{1, n}, \ldots, S_{n}^{j, n}$ together with the quantities $S_{n}^{n, k-1}, \ldots, S_{n}^{n, n+1}$. But these latter quantities also may be calculated from the Newton sums, since by rewriting (30) for the special case $j=n$, 


$$
S_{n}^{n, k}=\sum_{0 \leqslant j^{\prime} \leqslant n-1}(-1)^{j^{\prime}} S_{n}^{n, k-j^{\prime}-1} S_{n}^{n-j^{\prime}, n}
$$

we see that we may successively set $k=n+1, n+2, \ldots$, and recursively determine all the $S_{n}^{n, k}$ in terms of the Newton sums and $S_{n}^{n, n-1}=1$. This is done by the following

Algorithm. Given Newton sums $S_{n}^{1, n}, \ldots, S_{n}^{n, n}$ stored in $S N(1), \ldots, S N(n)$, respectively, to calculate the values $S_{n}^{n, n-1}=1.0, S_{n}^{n, n}=S N(n), S_{n}^{n, n+1}, \ldots$, $S^{n, n+K}$, and store them in $S K(1), \ldots, S K(K+2)$.

1. $S K(1) \leftarrow 1.0, S K(2) \longleftarrow S N(n), k \leftarrow n+1$.

2. $j^{\prime} \leftarrow 0$, SIGN $\longleftarrow 1.0, \alpha \longleftarrow k-n+2, S K(\alpha) \leftarrow 0.0$.

3. $S K(\alpha) \leftarrow S K(\alpha)+\operatorname{SIGN} * S N\left(n-j^{\prime}\right) * S K\left(\alpha-j^{\prime}-1\right)$.

4. If $k-j^{\prime} \neq n$ and $j^{\prime} \neq n-1$, SIGN $\leftarrow-\operatorname{SIGN}, j^{\prime} \leftarrow j^{\prime}+1$, go to 3 .

5. If $k \neq K, k \leftarrow k+1$, go to 2 .

6. Exit.

84 Dupont Street

Toronto, Ontario M5R 1V2, Canada

1. GASTON JULIA, "Mémoires sur l'itération des fonctions rationnelles," J. Math. Pures Appl., v. 1, 1918, pp. 47-245. 161-271.

2. P. FATOU, "Sur les équations fonctionnelles," Bull. Soc. Math. France, v. 47, 1919, pp.

3. PAUL MONTEL, Leçons sur les Familles Normales de Fonctions Analytiques et Leurs Applications, Gauthier-Villars, Paris, 1927.

4. PAUL MONTEL, "L'itération," Univ. Nac. La Plata Publ. Fac. Ci. Fisicomat. Serie Segunda Rev., v. 3, 1940, pp. 201-211.

5. H. RADSTRÖM, "On the iteration of analytic functions," Math. Scand., v. 1, 1953, pp. 85-92.

6. R. A. BROOKER, "The solution of algebraic equations on the EDSAC," Proc. Cambridge Philos. Soc., v. 48, 1952, pp. 255-270.

7. HUBERT CREMER, “Über die Iteration rationaler Funktionen,"Jber. Deutsch. Math.Verein., v. 9, 1952, pp. 185-210.

8. H. S. HALL \& S. R. KNIGHT, Higher Algebra, Macmillan, New York, 1950.

9. ERNST SCHRÖDER, "Über unendlich viele Algorithmen zur auflösung der Gleichungen," Math. Ann., v. 23, 1870, pp. 317-365.

10. A. S. HOUSEHOLDER, "Schröder and Trudi: a historical excursion," SIAM Rev., v. 16, 1974, pp. 344-348.

11. A. S. HOUSEHOLDER, Principles of Numerical Analysis, McGraw-Hill, New York, 1953.

12. JAMES L. HOWLAND, Private communication, 1977.

13. L. LEAU, "Étude sur les équations fonctionnelles," Ann. Fac. Sci. Univ. Toulouse, v. 11,1897 , pp. 22, 29-35.

14. E. DURAND, Solutions Numériques des Équations Algébriques. I, Masson, Paris, 1960.

15. P. JARRATT \& D. NUDDS, "The use of rational functions in the iterative solution of equations on a digital computer," Comput. J., v. 8, 1965, pp. 62-65. 